\title{
An Artificial Neural Network for Detection of Biological Early Brain Cancer
}

\author{
Mrs.Mamata S.Kalas, \\ M.Tech (CST), \\ Department Of IT, \\ Bharati Vidyapeeth's College Of Engg, \\ Kolhapur.Maharastra
}

\begin{abstract}
Human analysis on medical images is a difficult task due to very minute variations. Due to co-resemblance between affected \& original biological part \&due to larger data set for analysis. This makes the biological analysis for prediction of affects. The problem goes more complicated under cancer predictions basically in brain cancer. It is a challenging task to develop an automated recognition system which could process on a large information of patient and provide a correct estimation. So we are going to develop an automated cancer recognition system for MRI images. We implement the neuro fuzzy logic for the classification and estimation of cancer affect on given MRI image.
\end{abstract}

\section{Keywords:}

Co-resemblance, MRI images, Neuro fuzzy logic, Biological analysis.

\section{INTRODUCTION}

A technique in which the data from an image are digitized and various mathematical operations are applied to the data, generally with a digital computer, in order to create an enhanced image that is more useful or pleasing to a human observer, or to perform some of the interpretation and recognition tasks usually performed by humans. Also known as picture processing.

Manipulating data in the form of an image through several possible techniques. An image is usually interpreted as a twodimensional array of brightness values, and is most familiarly represented by such patterns as those of a photographic print, slide, television screen, or movie screen. An image can be processed optically, or digitally with a computer.

Image processing is an active area of research in such diverse fields as medicine, astronomy, microscopy, seismology, defense, industrial quality control, and the publication and entertainment industries. The concept of an image has expanded to include three-dimensional data sets (volume images), and even four-dimensional volume-time data sets. An example of the latter is a volume image of a beating heart, obtainable with x-ray computed tomography (CT). CT, PET, single-photon emission computed tomography (SPECT), MRI, ultrasound, SAR, confocal microscopy, scanning tunneling microscopy, atomic force microscopy, and other modalities have been developed to provide digitized images directly. Digital images are widely available from the Internet, CD-ROMs, and inexpensive charge-coupled-device (CCD) cameras, scanners, and frame grabbers. Software for manipulating images is also widely available. Image Processing is processing of the image so as to reveal the inner details of the image for further investigation. With the advent of digital computers.

Various areas in Image Processing :-

-Segmentation

$\bullet$ Enhancement

${ }^{\bullet}$ Compression

-Pattern recognition, etc.

Pattern recognition area is one of the evolving area for Biomedical applications.

\section{PROPOSED SYSTEM}

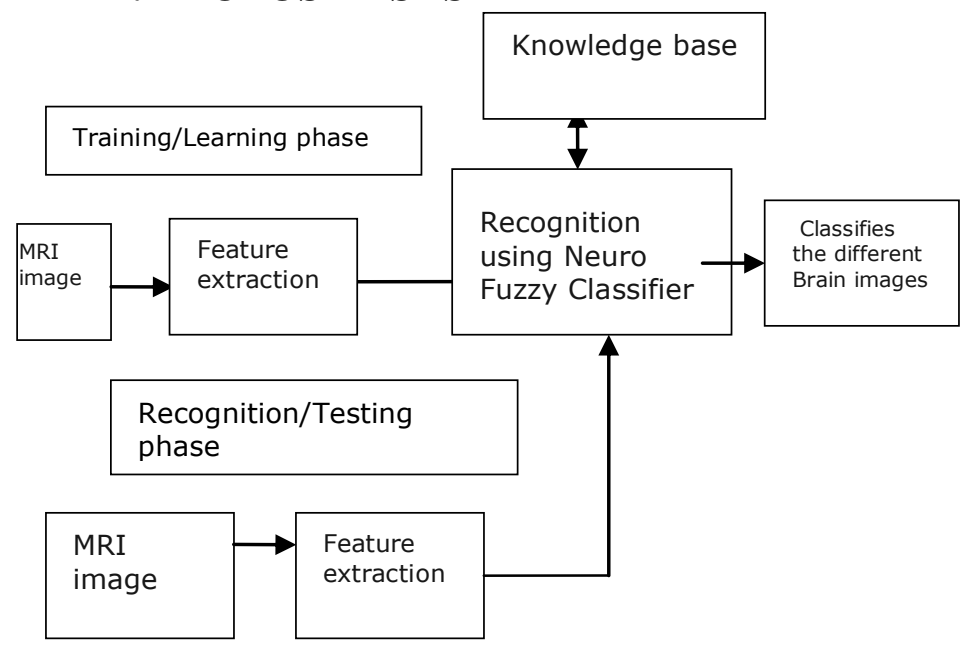

Figure 1.1: Proposed Methodology for Classification Of Brain Cancer.

\subsection{MRI Images}

Magnetic Resonance Imaging- Magnetic Resonance Image Segmentation .Segmentation of medical imagery is a challenging task due to the complexity of the images, as well as to the absence of models of the anatomy that fully capture the 
possible deformations in each structure. Brain tissue is a particularly complex structure, and its segmentation is an important step for derivation of computerized anatomical atlases, as well as pre- and intra-operative guidance for therapeutic intervention.

MRI segmentation has been proposed for a number of clinical investigations of varying complexity. Applications of MRI segmentation include the diagnosis of brain trauma where white matter lesions, a signature of traumatic brain injury, may potentially be identified in moderate and possibly mild cases. These methods, in turn, may require correlation of anatomical images with functional metrics to provide sensitive measurements of brain trauma. MRI segmentation methods have also been useful in the diagnostic imaging of multiple sclerosis], including the detection of lesions, and the quantization of lesion volume using multispectral methods. In order to understand the issues in medical image segmentation, in contrast with segmentation of, say, images of indoor environments, which are the kind of images with which general purpose visual segmentation systems deal, we need an understanding of the salient characteristics of medical imagery.

One application of our clustering algorithm is to map and identify important brain structures, which may be important in brain surgery. MRI is an imaging technique used in medical settings to produce high quality images, inside human body. It is more efficiently used in brain scanning.

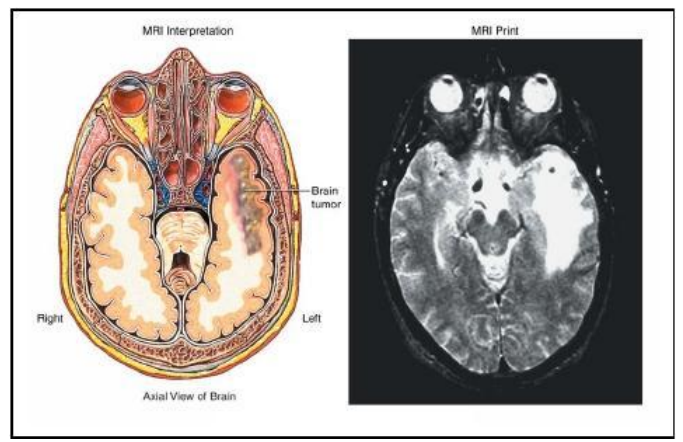

Figure 2. MRI Images

\section{Feature Extraction}

For the recognition of given query sample five invariant features are evaluated.

For the evaluation of these features, the image is processed through :

$\bullet$ Histogram Equalization

-Binarization

-Morphological Operations

$\bullet$ Region Isolation

$\bullet$ Feature Extraction

The above stated methods are used for both query images $\&$ the database images.

\subsection{Histogram Equalization}

Histogram equalization is the technique by which the dynamic range of the histogram of an image is increased. Histogram equalization assigns the intensity values of pixels in the input image such that the output image contains a uniform distribution of intensities. It improves contrast and the goal of histogram equalization is to obtain a uniform histogram. This technique can be used on a whole image or just on a part of an image. Histogram equalization redistributes intensity distributions. If the histogram of any image has many peaks and valleys, it will still have peaks and valley after equalization, but peaks and valley will be shifted. Because of this, "spreading" is a better term than "flattening" to describe histogram equalization. In histogram equalization, each pixel is assigned a new intensity value based on its previous intensity level.

\section{General Working}

The histogram equalization is operated on an image in three step:

\section{1). Histogram Formation}

2). New Intensity Values calculation for each Intensity Levels

3). Replace the previous Intensity values with the new intensity values.

i) The given MRI is equalized using histogram.

ii) The Histogram of an image represents the relative frequency of occurrences of pixel in a given image.
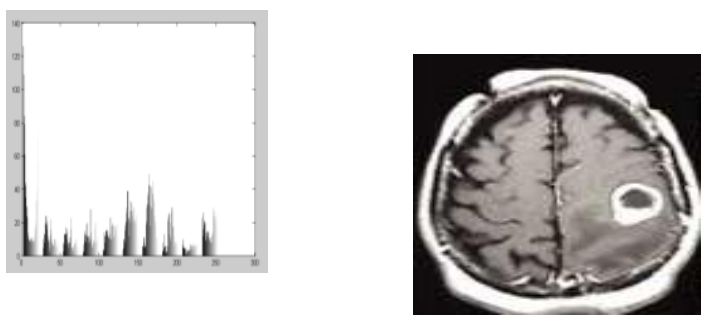

Fig 3.1 Histogram Equalized Image: The non-uniform varying image due to external conditions is equalized to a uniform variation.

\subsection{Binarization}

- Image binarization converts an image of up to 256 gray levels to a black and white image. Frequently, binarization is used as a pre-processor before OCR. In fact, most OCR packages on the market work only on bi-level (black \& white) images.

- The simplest way to use image binarization is to choose a threshold value, and classify all pixels with values above this threshold as white, and all other pixels as black. The problem then is how to select the correct threshold. In many cases, finding one threshold compatible to the entire image is very difficult, and in many cases even impossible. Therefore, adaptive image binarization is needed where an optimal threshold is chosen for each image area. 
For the equalized image the pixels are represented in a 0 to 255 gray level intensity.

- As the process is to extract the affected region or the accumulated region, a 2-level image representation would be sufficient for better computation.

• For the binarization of equalized image a thresholding method is used as illustrated :

Binarized Image bi,j $=255$ if $e(i, j)>T$

$$
\text { else } \quad b i, j=0
$$

where e(i,j) is the equalized MRI image and $\mathrm{T}$ is threshold derived for the equalized image.

${ }^{\bullet} \mathrm{M}$ is the masking matrix derived using neighbourhood estimation method, as given :

$$
\begin{aligned}
& \text { Let } e=p 1 \text { p2 p3 } \\
& \text { p4 p5 p6 } \\
& \text { p7 p8 p9 }
\end{aligned}
$$

be the equalized image matrix.Then the masking element $\mathrm{M}(\mathrm{p} 5)$ $=\max (|\mathrm{p} 4-\mathrm{p} 6|,|\mathrm{p} 2-\mathrm{p} 8|)$

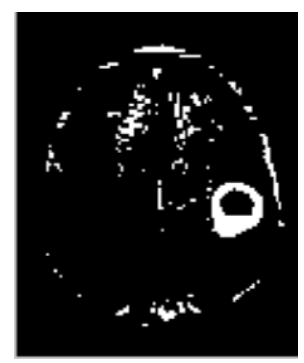

Figure 3.2 shows the binarized image for the given query image.

\subsection{Morphological Operations}

This is used as a image processing tools for sharpening the regions and filling the gaps for binarized image.The dilation operator is used for filling the broken gaps at the edges and to have continuities at the boundaries.A structuring element of $3 \times 3$ square matrix is used to perform dilation operation.

\section{Dilation and Erosion}

From these two Minkowski operations we define the fundamental mathematical morphology operations dilation and erosion:

Dilation -

$$
D(\mathrm{~A}, \mathrm{~B})=\mathrm{A} \oplus \mathrm{B}=\bigcup_{\beta \in \mathrm{B}}(\mathrm{A}+\beta)
$$

Erosion -

$$
E(\mathrm{~A}, \mathrm{~B})=\mathrm{A} \propto(-\mathrm{B})=\bigcap_{\beta \in \mathbb{B}}(\mathrm{A}-\beta)
$$

where $-\mathrm{B}=\{-\beta \mid \beta \in \mathrm{B}\}$

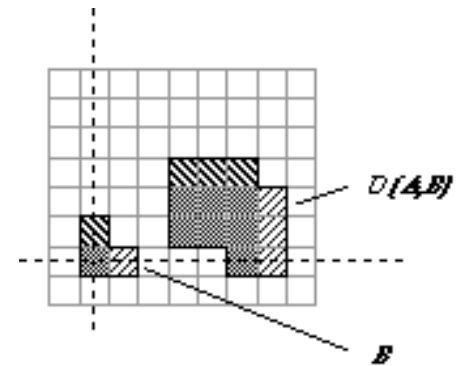

(a) Dilation $\mathrm{D}(A, B)($ b) Erosion $\mathrm{E}(A, B)$

Figure 3.3: A binary image containing two object sets $\boldsymbol{A}$ and $\boldsymbol{B}$. The three pixels in $\boldsymbol{B}$ are "color-coded" as is their effect in the result.

\section{Region Extraction}

It is required to display accurately the position relations of the extracted tumor and brain area from the MRI image which is used for the diagnosis of the brain tumor. We propose a method of extracting the brain tumor area using MRI images. In our method, after a base image is generated from one of slice image in MRI data, Region Growing method is applied to the selected slice image based on the base image. The area which is obtained by Region Growing method is considered as a new base image in the next step, and this extraction process is repeated for all slice image. Finally, we extracted the area of tumor and brain, and both are visualized in three-dimensional domain simultaneously to understand the position relations of the tumor. Onto the dilated image a filling operator is applied to fill the close contours. To filled image,centroids are calculated to localize the regions as shown beside. The final extracted region is then logically operated for extraction of Massive region in given MRI image.

\section{Feature Extraction}

To the extracted region the feature extraction process is applied for the calculation of 5 invariant features.

-Area

$\bullet$ Homoginiety

${ }^{\bullet}$ Contrast

- ASM( Angular second moment)

$\bullet$ Entropy

1. Contrast

$\mathrm{N}-1$

$f 1=\sum_{i, j=0} P i, j(i-j) 2$

2. Angular Second Moment (ASM)

$f 2=\sum_{i, j=0}^{N-1} P i, j$

3.Inverse Difference Moment (Homogeneity)

$f 3=\sum_{i, j=0}^{N-1} \underline{P i, j}$

4. Area 


\section{Entropy \\ $\mathrm{N}-1$ \\ $\mathrm{f5}=\sum_{\mathrm{i}, \mathrm{j}=0} \mathrm{Pi}, \mathrm{j}(-\ln \mathrm{Pi}, \mathrm{j})$}

The above mentioned process is applied on a clustered database consisting of 60 distinct MRI images categorized into 4 classes. The database classes are :

i) Gliomas class- Slow growing, with relatively well-defined borders.

ClassI
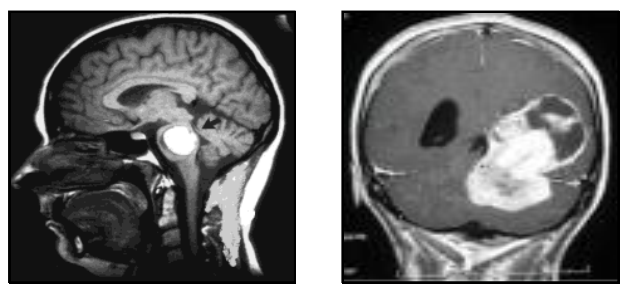

\section{Class II}
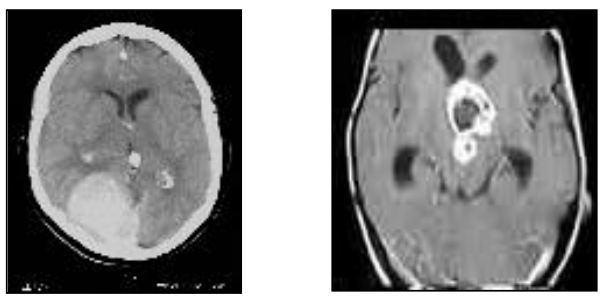

Classification

Fuzzy neural approach found to have more accurate decision making as compare to their counterparts.

The obtained features are processed using fuzzyfication layer before passing it to neural network.

\section{RESULTS}

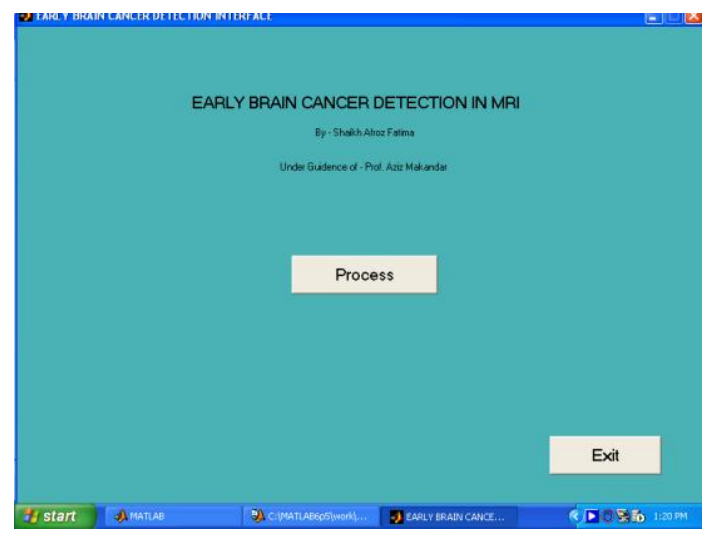

Fig 6.1 fig Initial GUI for Early Brain Cancer Detection System ii) Astrocytoma- Slow growing, Rarely spreads to other parts of the CNS , Borders not well defined .

iii) Anaplastic Astrocytoma Grows faster

iv) Glioblastoma multiforme (GBM) Most invasive type of tumor, Commonly spreads to nearby tissue, Grows rapidly.

Class III
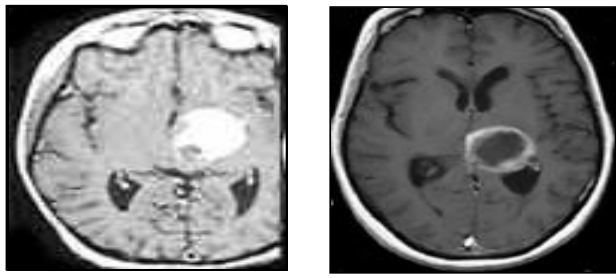

Class IV
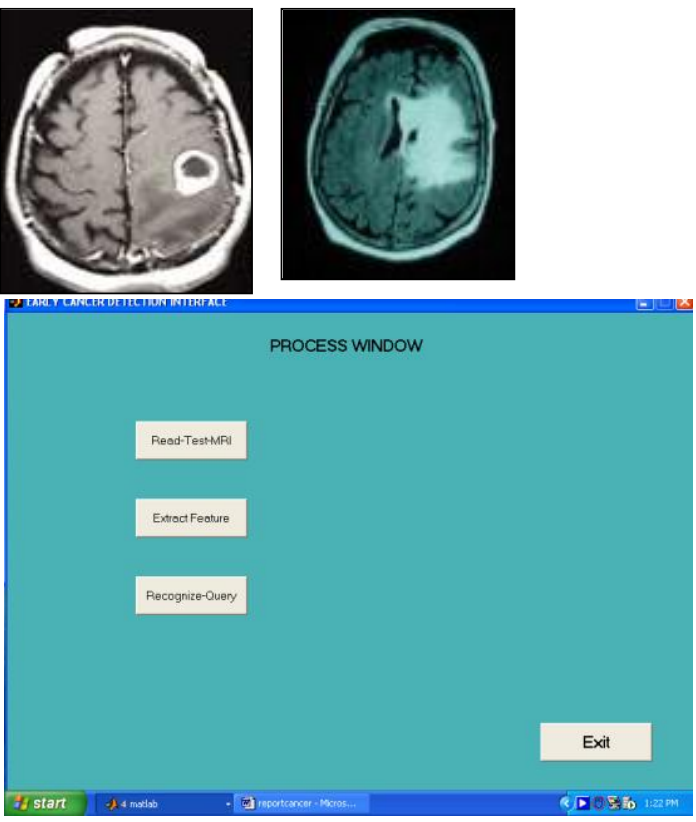

Fig 6.2 Process Window

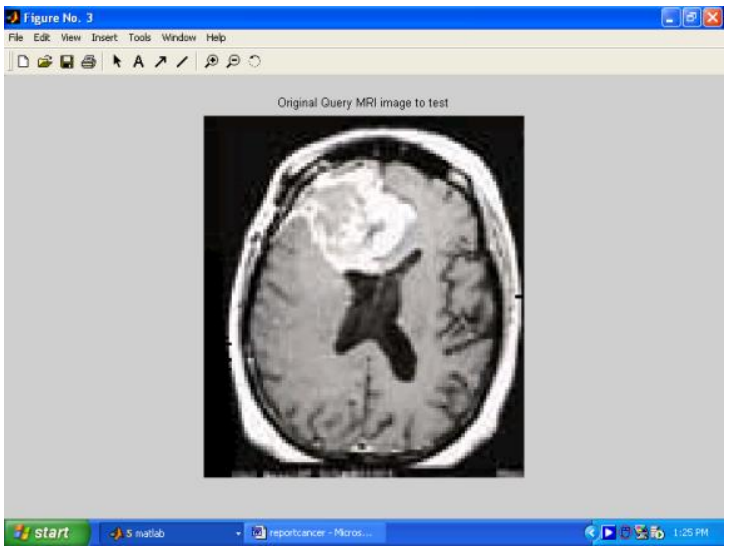

Fig 6.3 Original Input Image 


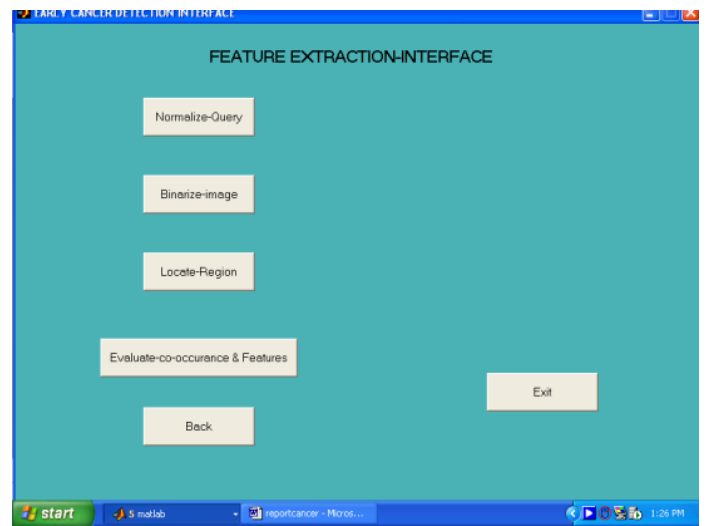

Fig 6.4 Feature Extraction Interface

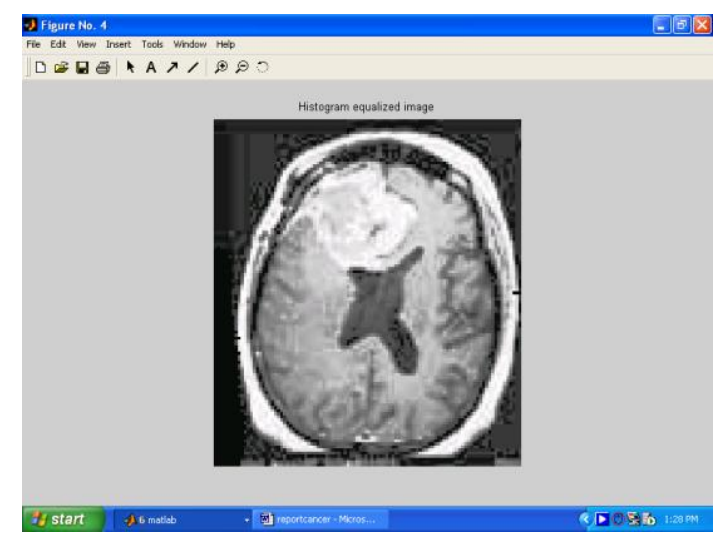

Fig 6.5 Histogram Equalized Image

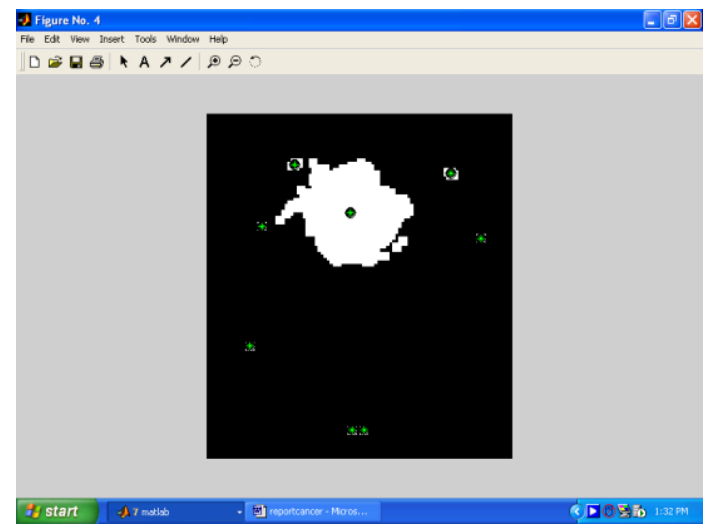

Fig 6.6 Intensity mapped Image

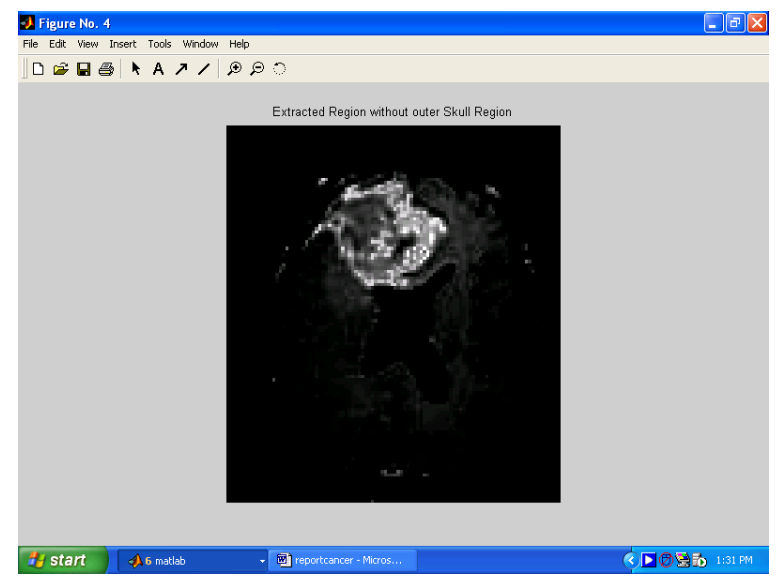

Fig 6.7 Morphological Operated dilated Image

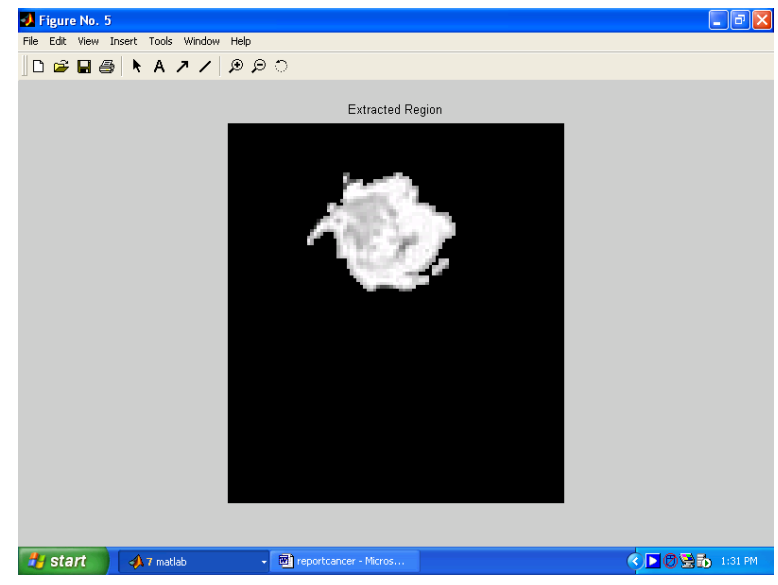

Fig 6.8 Extracted region with centroids

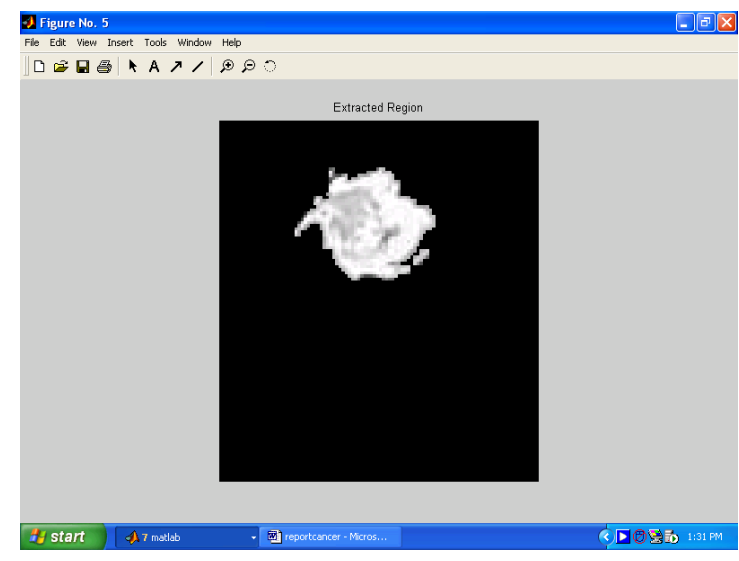

Fig 6.9 Extracted tumor region 


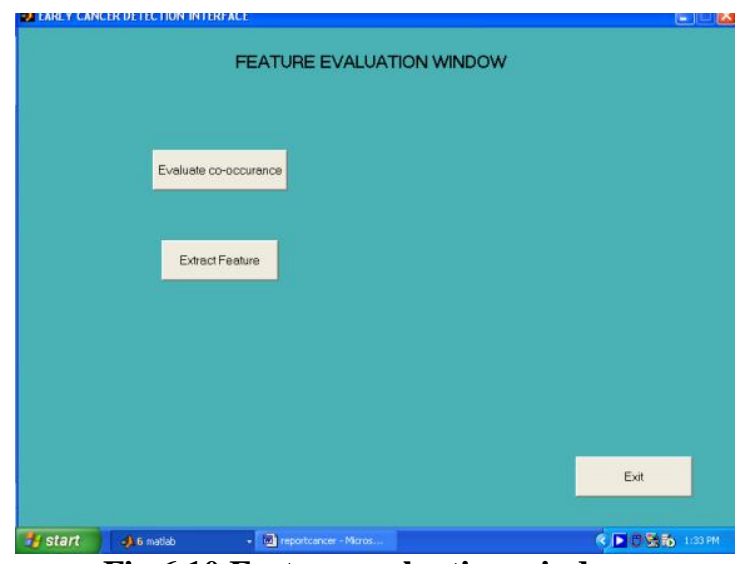

Fig 6.10 Feature evaluation window

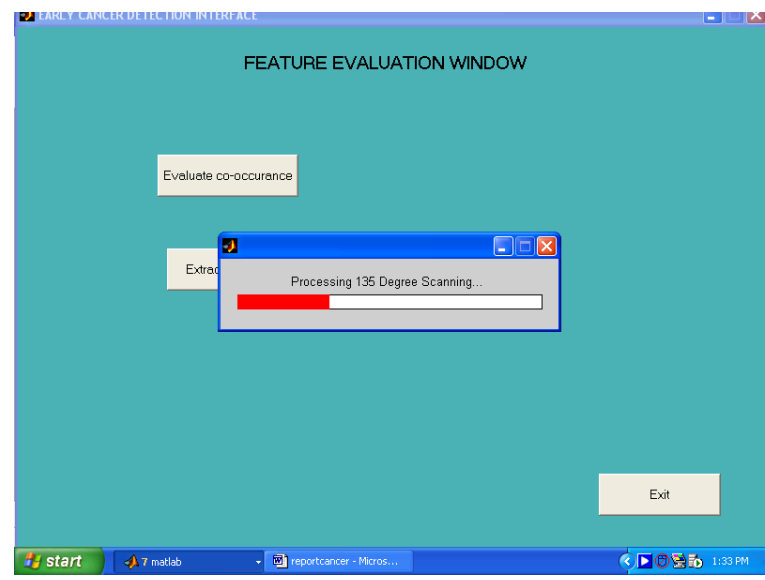

Fig 6.11 Processing MRI at different degrees

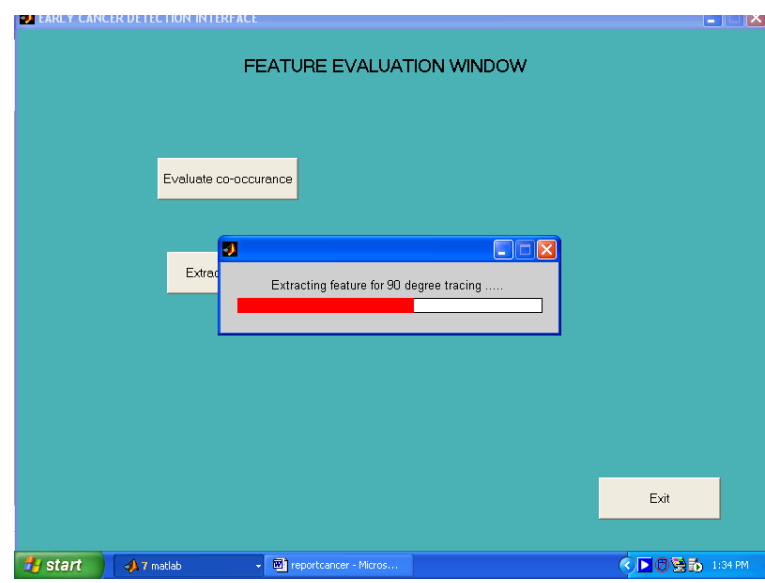

Fig 6.12 Extracting features of MRI image

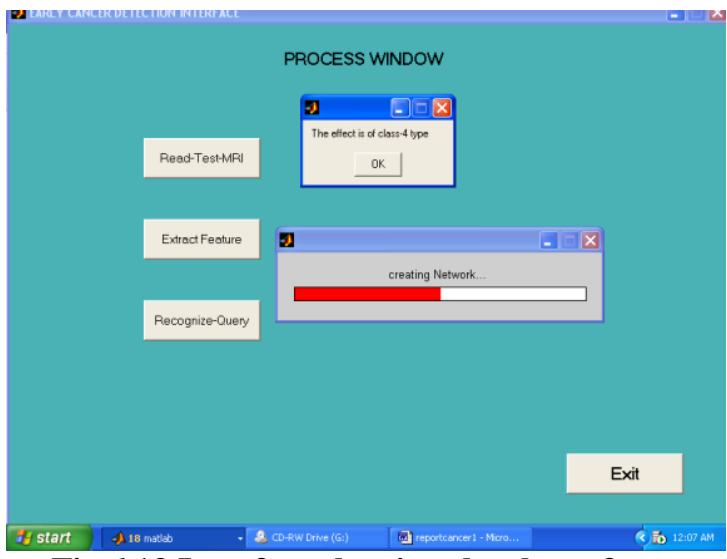

Fig 6.13 Interface showing the class of tumor

\section{CONCLUSION}

-This Research work project presents an automated recognition system for the MRI image using the neuro fuzzy logic. Texture features are used in the training of the neuro-fuzzy model. Cooccurrence matrices at different directions are calculated and Grey Level Co-occurrence Matrix (GLCM) features are extracted from the matrices.

-It is observed that the system result in better classification during the recognition process. The considerable iteration time and the accuracy level is found to be about $50-60 \%$ improved in recognition compared to the existing neuro classifier.

\section{REFERENCES}

[1] Sonka, M. Hlavac, V. Boyle, R. (2004). Image processing, Analysis, and Machine Vision, II Edition, Vikas Publishing House, New Delhi.

[2] Martin, H, Howard, D, and Mark, B. (2004). Neural Network Design. I Edition, Vikas Publishing House, New Delhi, India.

[3] Bose, N.K. and Liang, P. (2004). Neural Network Fundamentals with Graphs, Algorithms, and Applications, . I TMH Edition ,TMH, India.

[4] Gonzalez, R.C. Richard, E.W. (2004), Digital Image Processing, II Indian Edition, Pearson Education, New Delhi, India.

[5] Introduction to Neural Networks using Matlab 6.0 by S.N. Sivanandam, S. Sumathi, S.N. Deepa.

[6] Zhu H, Francis HY, Lam FK, Poon PWF. Deformable region model for locating the boundary of brain tumors. In: Proceedings of the IEEE 17th Annual Conference on Engineering in Medicine and Biology 1995. Montreal, Quebec, Canada: IEEE, 1995; 411

[7] T.K. Yin and N.T. Chiu, "A computer-aided diagnosis for locating abnormalities in bone scintigraphy by fuzzy system with a three-step minimization approach," IEEE Trans. Med. Imaging, vol.23, no.5, pp.639-654, 2004.

[8] X. Descombes, F. Kruggel, G. Wollny, and H.J. Gertz, "An object-based approach for detecting small brain lesions: Application to Virchow-robin spaces," IEEE Trans Med. Imaging, vol.23, no.2, pp.246-255, 2004.

[9] Cline HE, Lorensen E, Kikinis R, Jolesz F.Threedimensional segmentation of MR images of the head using 
probability and connectivity. J Computer Assist Tomography 1990; 14:1037-1045.

[10] Vannier MW, Butterfield RL, Rickman DL, Jordan DM, Murphy WA, Biondetti PR. Multispectral magnetic resonance image analysis. Radiology 1985; 154:221-224.

[11] A Dramatic Evolution in seeing \& treating tumorsFeatured Article by Jonnie Rohrer from Visions, Fall/Winter 2004

[12] Medicine at Michigan - Kara Gavin

[13] Novel Genetic Technology to Predict Treatment for Brain Cancer Timothy Cloughesy, M.D., UCLA assistant professor and neurologist Spring 2000

[14] University of Alberta Brain Tumor Growth Project: Automated Tumor Segmentation.
[15] Primary Brain Tumors in the United States Statistical Report 2002-2003. Central Brain Tumor Registry of the United States (CBTRUS)

[16] Surgical Brain Cancer Pictures, Brain MRI photos.19972005 CaringBridge, a non-profit organization.

[17] Cigdem, D and Bullent, Y. (2004). Automatic cancer diagnosis based on histopathological images: a systematic survey. Technical Report, Rensselaer polytechnic institute, Dept. of computer science, TR-05-09.

[18] Astrocytomas By Patrick J. Kelly, M.D., FACS, Professor and Chairman of Neurosurgery June 25, 1995( Grades of Tumor)

[19] Gibbs P, Buckley DL, Blackband SJ, Horsman A. Tumor volume determination from MR images by morphological segmentation. Phys Med Biol 1996; 41:2437-2446. 